\title{
Diabetic wound infection: A review on microbial population and infection control
}

\author{
Nur Amiera Syuhada Rozman ${ }^{\mathrm{a}}$, Woei Yenn Tong ${ }^{\mathrm{a}^{*}}$, Chean Ring Leong ${ }^{\mathrm{a}}$, Wen-Nee Tan ${ }^{\mathrm{b}}$, \\ Syarifah Ab Rashid ${ }^{a}$ \\ ${ }^{a}$ Universiti Kuala Lumpur, Branch Campus Malaysian Institute of Chemical and Engineering Technology, Lot 1988 Kawasan Perindustrian \\ Bandar Vendor, Taboh Naning, 78000 Alor Gajah, Melaka \\ ${ }^{b}$ Chemistry Section, School of Distance Education, Universiti Sains Malaysia, 11800 Gelugor, Pulau Pinang
}

Received 27th March 2020 / Accepted 17th June 2020

\begin{abstract}
A diabetic foot ulcer is one of the major complications of diabetes and it leads to lower extremity amputation in patients. This review explores the current research on microbial populations on diabetic wounds, and also treatment alternatives to combat the infection on chronic diabetic wounds. The microbial communities exist in diabetic wound infection are diverse. Microbes rarely survived in single species of planktonic cells. They usually exist in a complex polymicrobial biofilm population which consists of different types of microorganisms. Furthermore, the development of bacterial biofilm on the wound that usually consists of multidrug-resistant pathogens also delayed the wound healing. To overcome this problem, many types of modern wound dressing were developed including hydrocolloid, hydrogel, alginate and collagen wound dressing. Besides, modern biotechnological advancements such as cell therapy, bioengineered skin, dermal scaffolds, tissue-engineered artificial skin and growth factors were also employed to promote the recovery of the wound. In conclusion, diabetes mellitus is a major health care challenge worldwide. Diabetic patients are at risk for developing foot ulcer which ultimately leads to amputation; hence a safe and effective alternative treatment is required to improve diabetic patients' quality life.
\end{abstract}

Keywords: Diabetic wound, infection control, microbial population

\section{INTRODUCTION}

Diabetic foot ulcer is described as ulceration, infection and destruction of deep tissues, in a foot of person associated with neurological abnormalities and peripheral vascular disease in the lower limb (Van Netten et al., 2019). Diabetic foot ulcer is one of the major complications of diabetes and it possibly leads to lower extremity amputation in patients. Furthermore, a study conducted from 2007 to 2008 in China reported that the prevalence of foot ulcer was 92.4 million for diabetes and 148.2 million for pre-diabetes
(Yang et al., 2010). About 24\% of diabetes patients underwent amputation within the first year after being affected with foot ulcers. This can be accessed via physical inspection such as the skin of vascular, neurological, and musculoskeletal systems (Alexiadou and Doupis, 2012). It is important to identify the early clinical signs of vascular damage in the limbs before ulcer formation such as atrophic foot, lack of hair growth on the dorsum, cool temperature of the limb, thin shining and atrophic skin (Ahmad,

*Author for correspondence: Woei Yenn Tong, Universiti Kuala Lumpur, Branch Campus Malaysian Institute of Chemical and Engineering Technology, Lot 1988 Kawasan Perindustrian Bandar Vendor, Taboh Naning, 78000 Alor Gajah, Melaka. Email - wytong@unikl.edu.my 
2016). Microbial infections become a major issue that may affect deep tissue, joints and bones (Noor et al., 2017). The early stage of diabetic foot ulcer formation is the loss of sensation due to peripheral neuropathy factor (Pallela and Narahari, 2017). Due to the loss of sensation, the repetitive minor injuries may eventually lead to foot ulcer (Alexiadou and Doupis, 2012). Furthermore, the ulcers are prone to the colonization of various pathogenic microorganisms (Weledji and Fokam, 2014).

One of the issues associated with a diabetic ulcer is the expensive medical treatment cost. The total expenses on wound management products have increased by $4.6 \%$ annually to 28.7 billion USD in 2013. Diabetic patients with foot ulcer showed a 150-fold increased risk of amputation, which is always associated with poor wound management (Pednekar et al., 2015). On the other hand, diabetic foot ulcer requires an intensive multidisciplinary assessment that usually handled by experts like nurses, doctors and specialists. Proper management of the ulcer may contribute to the avoidance of wound infection. A good wound management system significantly lowered the risk of amputation by simple practice on daily basis such as good footwear, chiropody, cleanliness, aggressive surgical radical debridement, off-loading, and ulcer management (Ahmad, 2016).

\section{Diabetic wound infection}

High bioburden delays the healing of infected wounds. The development of wound infection is an ongoing problem among diabetic patients. Usually, diabetic patients are more susceptible to develop infections as the immune system was diminished. Furthermore, severe wound infections also increase the risk of lower extremity amputations. Thus, wound infection causes prolonged hospitalization, thereby increasing the cost of the treatment (Tipton et al., 2019). There are several classic clinical signs of infections including erythema, localized warmth around the site of wound, edema, purulence exudate and fever (Green, 2012). Proper care practices are necessary to prevent morbidity, especially amputations.

Generally, the wounds are easily infected once they are exposed to the colonization of pathogenic microorganism. Wound colonization refers to the multiplying of various microbial species on a wound (Tipton et al., 2019). This leads to wound infection due to the apparent microbial growth and colonization. Staphylococcus aureus is the most common bacterium found in a diabetic wound that leads to the alteration of wound healing (Gardner et al., 2013). Hirsch et al. (2008) performed a study of wound healing on animal model inoculated with $S$. aureus. The results showed that $S$. aureus significantly impaired the wound healing of diabetic wound and the infection also promotes invasive co-infection of other bacteria.

\section{Microbial species on diabetic wound}

The microbial communities exist in diabetic wound infection are diverse. Microbes rarely survived in single species of planktonic cells. They usually exist in a complex polymicrobial biofilm population which is consist of different types of microorganisms (Peters et al., 2012). The common bacteria in wound are Pseudomonas aeruginosa, Acinetobacter baumannii, Stapbylococcus aureus, Enterococcus faecalis, Escherichia coli, Klebsiella pneumoniae and Enterobacter spp. (Hirsch et al., 2008). S. aureus and P. aeruginosa are the most common bacteria isolated from chronic wound (Fazli et al., 2009; Serra et al., 2015). Numerous studies have been conducted to study the role of multi-species pathogenic biofilms related to chronic diabetic wound before determining an effective method for wound infection treatment (Dalton et al., 2011; Tipton et al., 2020; Wu et al., 2019). Various methods have been employed to identify the bacterial population in the chronic wound such as qualitative measures, quantitative measures, polymerase chain reaction, and metagenomics methods (Martin et al., 2010; Wu et al., 2019). However, these techniques require a substantial amount of repetition to obtain accurate results. To overcome this problem, newly developed techniques such as pyrosequencing, microarrays and next generation sequencing were used (Wu et al., 2019). In such, a study was performed by Dowd et al. (2008) to determine the bacterial diversity in the chronic wound by utilizing pyrosequencing, denaturing gradient gel electrophoresis, and full ribosome shotgun sequencing. The study revealed that different bacterial populations were identified in 
different types of wound which majored by $62 \%$ of an obligate anaerobic microorganism.

The obligate anaerobic bacteria found in diabetic foot ulcer are Peptostreptococcus, Prevotella, Finegoldia and Peptoniphilus (Howell-Jones et al., 2005). Granov et al. (2019) reported that about 74 to $95 \%$ anaerobic bacteria found in the site of the infected wound were Peptostreptococcus and Bacteroides. Although the prevalence of anaerobic bacteria is less, it is still the cause of infections (Van Netten et al., 2019). In contrast, Senneville et al. (2006) reported that anaerobic bacteria play a minor role in diabetic wound infection. Dezfulian et al. (2011) reported that $S$. aureus was the most frequently isolated bacteria from a patient with diabetic foot infections in Iranian Hospital. Furthermore, the finding is supported by Gonzalez et al. (2003) where they reported S. aureus was the predominant bacteria found in the infection site followed by Enterococcus, Streptococcus, and Enterobacteriaceaes (Gonzalez et al., 2003).

In fact, $S$. aureus has been found as an opportunistic pathogen that able to adapt easily in different environmental conditions (Nair et al., 2014). The most commonly isolated bacteria from diabetic foot infection were $P$. aeruginosa contributing about $(35 \%)$ followed by $S$. aureus $(19 \%)$ and MRSA (6\%) (Parsa and Samani, 2015). $P$. aeruginosa develops acquired antibiotic resistance due to its ability to secrete virulence factors such as pili, type III secretion systems, quorum-sensing molecules, extracellular products like pyocyanin, and polysaccharides (Sadikot et al., 2005). Most $P$. aeruginosa strains secrete pyocyanin (N-methyl-1-hydroxyphenazine), a blue redoxactive secondary metabolite with ability to induced production of hydrogen peroxide in both eukaryotic and prokaryotic cells (Lau et al., 2004). This process causes cell death due to oxygen reduction. Colvin et al. (2012) stated that $P$. aeruginosa produced at least three types of polysaccharides to form biofilm. These polysaccharides form strong biofilm structure that protect them from the antimicrobial agents. Biofilm of $S$. aureus and $P$. aeruginosa have been well studied. The interaction of polymicrobial infections between these two microorganisms have been derived from both in vitro and in vivo studies. Besides, the conflict of $S$. aureus and $P$. aeruginosa also resulted in a chronic infection that can impair the wound healing process and promote antibiotic resistance (Colvin et al., 2012; Short et al., 2014). In agreement, Pastar et al. (2013) demonstrated the interactive effect of MRSA and $P$. aeruginosa by utilizing porcine wound healing model. The co-infection works well in slowing down the healing process and limits the antibiotic response. This implication significantly indicates the synergistic effect of both pathogenic species. In the following years, Serra et al. (2015) published a review on the role of $S$. aureus and $P$. aeruginosa in a chronic wound. They observed that these most common bacteria isolated from chronic wound exhibit a few virulence factors which make them acquired antibiotic resistance.

\section{Biofilm in diabetic wound}

Biofilm is developed when any group of microorganisms embed to each other and form a matrix of extracellular polymeric substance (Wei et al., 2019). The development of bacterial biofilm usually consists of multidrug-resistant pathogens that cause hospital-acquired infection. It has been reported that different microbial species were found on wound biofilm (Elias and Banin, 2012). Besides, according to the National Institute of Health, 65 to $80 \%$ of chronic infections are associated with biofilm formation including oral soft tissues, dental implants, urinary tract prostheses, and lung tissues. Since then, various strategies have been employed to prevent and eradicate the biofilm formation. Majorities of microbial biofilm studies in the last decade were based on in vitro models such as microtiter plate and continuous flow chamber/reactor (Fabrega et al., 2009; Durmus et al., 2012). Although limited evidence was collected to comprehend the biofilm formation using these techniques, the outcomes of the studies provide important information on biofilm survival, eradication and mechanisms of microbial cell in biofilm development.

Extracellular polysaccharides are the main components in the biofilm matrix. These polysaccharides help the bacteria to adhere to a host cell and protect them from environmental stress (Limoli et al., 2015). Bacterial biofilms delay the diabetic wound healing and it is also crucial to demonstrate the role of biofilms in delaying the healing of the wound (Percival et al., 2015). These polysaccharides block complement activation, depress the lymphoproliferative response and prevent opsonin detection on bacterial cell wall by 
phagocytes, hence the wound recovery is delayed (Bunyan et al., 2019). Besides, biofilm also limits the leukocytes to the wound and attenuates their ability to produce antimicrobial reactive oxygen species (Campoccia et al., 2019). More importantly, the exopolysaccharides in the biofilm serves as a physical barrier, which is a cause of antibiotic resistance. The polysaccharides slow down the diffusion of antibiotic to the wound (Bunyan et al., 2019). Various studies were conducted to investigate the wound associated with diabetic patients. Several in vivo models were used for biofilm study. The formation of biofilm delayed the wound healing of diabetic rats infected with $P$. aeruginosa (Zhao et al., 2010).

Biofilm is a unique community where different types of microorganisms work synergistically in forming a structure. Numerous stages of biofilm development were reported, including initial attachment, irreversible attachment, maturation I, maturation II and dispersion (Monroe, 2007). Initially, the development of biofilm begins with the freefloating of microbial cells at the wound surface. At this stage, the movement of the bacterial communities was driven by flagella and type IV pili that promote the colonization of microcolony against subtracting (Conrad, 2012). The planktonic cells swim along the surface in Brown motion. Colonization of bacteria communities is formed via Van der Waals interaction between the outer cell wall of bacterial cell with the surface (Renner and Weibel. 2011). Then, this situation leads to the development of the matured threedimensional structure of biofilm followed by dispersion (Renner and Weibel, 2011). During colonization, the communities of the cells can communicate with each other using a chemical signal called quorum sensing which also coordinates the gene expression (Geske et al., 2005). Quorum sensing is a mechanism where the cells produce and deliver their signals called autoinducers to increase the cell density (Hense and Schuster, 2015). Thus, quorum sensing is another factor contributing to antimicrobial-resistant and it accelerates the growth of biofilm (Flickinger et al., 2011).

Furthermore, biofilms can grow virtually in any surfaces with several requirements. Environmental factors including $\mathrm{pH}$, temperature, rheological as well as adhesive properties, need to be considered in biofilm formation (Garrett et al., 2008). Basically, the biofilm is actually an adaption of the microbial cells with the environmental conditions that vary on several stresses (Zhang et al., 2013). O'toole et al. (2000) discovered that genes and regulatory circuits are important mechanisms for initial cellsurface interaction, biofilm maturation and the return of biofilm microorganisms to a planktonic mode of growth. Antibiotic treatment of diabetic wound with biofilm formation has always been challenging. The efficacy of systemic antibiotic therapy was deduced by 25 to $20 \%$ with the biofilm formation (Wei et al., 2019). Besides, the drug resistance of bacteria can be increased to 1000 to 1500 times after biofilm formation, compared to the planktonic cells (Mendoza et al., 2019). Antibiotic resistance of wound bacteria has a significant influence on wound healing among diabetic patients.

\section{Infection control}

Wound dressing is one of the primary treatments against foot ulcer. The dressing is designed to maintain moisture, promote wound healing, lower the risk of scar formation and protect the wound from any other impairments (Moura et al., 2013). Traditionally, bandages, plaster, lint and gauze were used in wound management. However, traditional wound dressing failed to provide a moist environment and maintain the $\mathrm{pH}$ of wound sites (Boateng et al., 2008). To overcome this problem, many types of modern wound dressing were developed include hydrocolloid, hydrogel, alginate and collagen wound dressing (Dhivya et al., 2015; Weller et al., 2020). Hydrocolloid dressings are composed of hydrocolloid materials such as gelatin, pectin, and carboxymethyl cellulose which provide a moist environment for wound healing and barrier to external microorganisms (Dorner et al., 2009). In addition, hydrocolloid dressings are able to absorb a large amount of wound fluid and exudates for a sustained period. Thus, the frequency of dressing changes can be reduced (Hilton et al., 2004). Due to its oxygen impermeable nature, the dressing increases the rate of collagen synthesis during wound recovery (Oliveira et al., 2020).

Hydrogel dressing is commonly used for necrotic, sloughy, granulating, epithelializing wounds (Moody, 2006). It is designed in a 
hydrophilic polymer matrix which made up from synthetic polymer like poly (methacrylates) and polyvinyl pyrrolidine with high water content (Dhivya et al., 2015). It is suitable for all four stages of wound. Dumville et al. (2013) conducted a study by using hydrogel wound dressing on a diabetic foot ulcer. The study showed that hydrogel dressing was more effective than basic wound dressing on diabetic foot ulcer and there is no statistical difference in a number of heal ulcer with larval therapy, platelet-derived growth factor, and a different brand of hydrogel dressing. In order to meet a high-quality wound dressing, researchers have also exploited smart polymers to cooperate with hydrogel. For instance, $N$ isopropylacrylamid (NIPAM), a thermoresponsive drug carrier is grafted within the hydrogel layer in dermal patches. The substance becomes hydrophilic at a temperature of $32^{\circ} \mathrm{C}$ and below, but hydrophobic at the higher temperature. This condition can be activated through copolymerization for optimal drug release (Wei et al., 2009). The charge of the hydrogel materials can be also modified to control the release of the drug to the wound, thus can frequency of dressing changing can be reduced (Venault et al., 2019).

Alginate dressing is another type of modern dressing used in direct contact with a wound. Alginate is a natural polymer derived from brown seaweed (Hilton et al., 2004). Alginate has a higher absorbency compared to normal dressing where it can absorb 20 times of their weights which make them effective to be used for mild to heavy exudate (Baker, 2005). A controlled random trial performed by Lalau et al. (2002) showed that alginate wound dressing improved the wound healing of diabetic patients compared to normal dressing. However, alginate dressing displays a few disadvantages that limit the usage; it may be painful for a patient with small amount of exudate since it requires a large moisture content to function effectively and it always require a secondary dressing which will increase the cost of dressing changes (Dabiri et al., 2016). Furthermore, there is no evidence on the effectiveness of alginate dressing in healing diabetic foot ulcer (Dumville et al., 2013). Latest advanced wound dressing was made up of collagen. Due to its chemotactic properties, it stimulates new tissue growth by migrating cells like fibroblasts and keratinocytes (Fleck and Simman, 2010). However, collagen dressing triggers an allergic response in some patients (Chattopadhyay and Raines, 2014). Recently, a clinical study by Djavid et al. (2020) also revealed that the usage of collagen matrix dressing significantly accelerated the healing to hard-toheal diabetic foot ulcers.

Recently, various types of antimicrobial agents, nanoparticles and natural products have been incorporated into the wound dressing. Unfortunately, the choice of antimicrobial agent showed no clear evidence if one better than others in treating diabetic food ulcer because the data from previous reports were heterogeneous (Selva et al., 2015). The inappropriate and indiscriminate use of antibiotics is a key reason contributing to antibiotic resistance in microorganisms. Silver finished cottons are widely used in wound care products. In addition, an in vivo study conducted by Kostenko et al. (2010) on animals mimic the condition found in a bed wound showed that silver-based wound dressing exhibited a significant reduction number of biofilm viable cells in 7 days treatment. Kalishwaralal et al. (2010) reported that silver nanoparticles eradicate the biofilm production in $P$. aeruginosa and $S$. epidermis by $98 \%$. Despite of this, silver-finished product could lead to increased morbidity with chronic ingestions. Moreover, inhalation of heavy metal preparations also leads to deposition of heavy metal particles in the skin, eyes, kidneys and livers (Rovira et al., 2015). In other words, due to the exposure or ingestion of excessive silver element, the skin discoloration in the wound area or argyria appeared (Baker et al., 2007). This skin staining usually remained visible after treatment (Zweiker et al., 2014). For the silver ion to function as an effective bactericidal agent, it needs to reach a concentration of 30-40 ppm (Murphy and Evans, 2012). However, as recorded by Poon and Burd (2004), the silver ion happens to be toxic to keratinocytes and fibroblasts at $33 \mathrm{ppm}$ and above, especially. There are a thousand types of wound dressing available in last decades in different shapes, size and color. However, it is a challenging task for clinicians to find the most appropriate wound dressing for different types of wound as no single wound dressing is suitable for all wound treatment. 
Many advanced technologies were developed to help the recovery of wound, these modern therapies including cell therapy, bioengineered skin, dermal scaffolds, tissue-engineered artificial skin and growth factors (Debels et al., 2015; Falanga, 2020; Frueh et al., 2017). However, they are expensive and not easy to use. Negative pressure wound is another therapy that may represent a treatment that uses vacuum to remove access wound fluid that usually enhances and promotes wound healing (Bovill et al., 2008). However, the results were inconsistent. A comparative study by Ravari et al. (2013) demonstrated that vacuum-assisted closure displayed a significant improvement of the wound after 2 weeks with no single amputation case was reported. However, moist wound dressing did not show significant improvement in the recovery of diabetic foot ulcer. Besides, tissue engineering can be applied to substitute the damaged skin and to improve new tissues regeneration. The artificial skin is highly useful for treating chronic wounds; however, this method is highly expensive (Langer and Rogowski, 2009).

\section{CONCLUSION}

In conclusion, diabetes mellitus is a major health care challenge worldwide. Clinically, diabetic patients are at risk for developing foot ulcer which ultimately leads to amputation. Besides, wound healing in diabetic patients is frequently impaired where the delay in healing time is commonly associated with microbial infection. Due to this, a safe and effective alternative treatment is required to improve diabetic patients' quality life.

\section{REFERENCES}

Ahmad, J. 2016. The diabetic foot. Diabetes and Metabolic Syndrome: Clinical Research and Reviews 10(1): 48-60.

Alexiadou, K. \& Doupis, J. 2012. Management of diabetic foot ulcers. Diabetes Therapy 3(1): 4-19.

Baker, C. D., Federico, M. J., \& Accurso, F. J. 2007. Case report: skin discoloration following administration of colloidal silver in cystic fibrosis. Current Opinion in Pediatrics 19(6): 733-735.

Baker, P. D. 2005. Creating the optimal environment. An overview of dressings for chronic wounds. Advance for Nurse Practitioners 13(7): 37-38.
Boateng, J. S., Matthews, K. H., Stevens, H. N., \& Eccleston, G. M. 2008. Wound healing dressings and drug delivery systems: a review. Journal of Pharmacentical Sciences 97(8): 2892-2923.

Bovill, E., Banwell, P. E., Teot, L., Eriksson, E., Song, C., Mahoney, J., \& Whitworth, I. 2008. Topical negative pressure wound therapy: a review of its role and guidelines for its use in the management of acute wounds. International Wound Journal 5(4): 511-529.

Bunyan, I. A., Hadi, O. M., \& Al-Mansoori, H. A. 2019. Phenotypic detection and biofilm formation among Pseudomonas aeruginosa isolated from different sites of infection. International Journal of Pharmaceutical Quality Assurance 10(02): 334-341.

Campoccia, D., Mirzaei, R., Montanaro, L., \& Arciola, C. R. 2019. Hijacking of immune defences by biofilms: a multifront strategy. Biofouling 35(10): 1055-1074.

Chattopadhyay, S., \& Raines, R. T. 2014. Review collagen-based biomaterials for wound healing. Biopolymers 101(8): 821-833.

Colvin, K. M., Irie, Y., Tart, C. S., Urbano, R., Whitney, J. C., Ryder, C., \& Parsek, M. R. 2012. The Pel and Psl polysaccharides provide Pseudomonas aeruginosa structural redundancy within the biofilm matrix. Environmental Microbiology 14(8): 1913-1928.

Conrad, J. C. 2012. Physics of bacterial near-surface motility using flagella and type IV pili: implications for biofilm formation. Research in Microbiology 163(9-10): 619-629.

Debels, H., Hamdi, M., Abberton, K., \& Morrison, W. 2015. Dermal matrices and bioengineered skin substitutes: a critical review of current options. Plastic and Reconstructive Surgery Global Open 3(1): e284.

Dabiri, G., Damstetter, E., \& Phillips, T. 2016. Choosing a wound dressing based on common wound characteristics. Advances in Wound Care 5(1): 32-41.

Dezfulian, A., Salehian, M. T., Amini, V., Dabiri, H., Azimi Rad, M., Aslani, M. M., \& Zali, M. R. 2011. Bacteriological study of diabetic foot infections in an Iranian hospital. Iranian Red Crescent Medical Journal 13(8): 590-591.

Djavid, G. E., Tabaie, S. M., Tajali, S. B., Totounchi, M., Farhoud, A., Fateh, M., \& Taghizadeh, S. 2020. Application of a collagen matrix dressing on a neuropathic diabetic foot ulcer: a randomised control trial. Journal of Wound Care 29(3): 13-18.

Dhivya, S., Padma, V. V., \& Santhini, E. 2015. Wound dressingsa review. BioMedicine 5(4): 24-28.

Dorner, B., Posthauer, M. E., \& Thomas, D. 2009. The role of nutrition in pressure ulcer prevention and treatment: National pressure ulcer advisory panel white paper. Advances in Skin and Wound Care 22(5): 212-221.

Dowd, S. E., Sun, Y., Secor, P. R., Rhoads, D. D., Wolcott, B. M., James, G. A., \& Wolcott, R. D. 2008. Survey of bacterial diversity in chronic wounds using pyrosequencing, DGGE, and full ribosome shotgun sequencing. BioMedical Central Microbiology 8(1): 43-58.

Dumville, J. C., O’Meara, S., Deshpande, S., \& Speak, K. 2013. Hydrogel dressings for healing diabetic foot ulcers. Cochrane Database System Review 12(7): 1-13.

Durmus, N. G., Taylor, E. N., Inci, F., Kummer, K. M., Tarquinio, K. M., \& Webster, T. J. 2012. Fructose-enhanced reduction of bacterial growth on nanorough surfaces. International Journal of Nanomedicine 7: 537-545.

Elias, S., \& Banin, E. 2012. Multi-species biofilms: living with friendly neighbors. Federation of European Microbiological Societies Microbiology Reviews 36(5): 990-1004.

Fabrega, J., Renshaw, J. C., \& Lead, J. R. 2009. Interactions of silver nanoparticles with Pseudomonas putida biofilms. Environmental Science and Technology 43(23): $9004-$ 9009. 
Fazli, M., Bjarnsholt, T., Kirketerp-Moller, K., Jørgensen, B., Andersen, A. S., Krogfelt, K. A., \& Tolker-Nielsen, T. 2009. Nonrandom distribution of Pseudomonas aeruginosa and Staphylococcus aureus in chronic wounds. Journal of Clinical Microbiology 47(12): 4084-4089.

Fleck, C. A., \& Simman, R. 2010. Modern collagen wound dressings: function and purpose. The Journal of the American College of Certified Wound Specialists 2(3): 50-54.

Flickinger, S. T., Copeland, M. F., Downes, E. M., Braasch, A. T., Tuson, H. H., Eun, Y. J., \& Weibel, D. B. 2011. Quorum sensing between Pseudomonas aeruginosa biofilms accelerates cell growth. Journal of the American Chemical Society 133(15): 5966-5975.

Frueh, F. S., Menger, M. D., Lindenblatt, N., Giovanoli, P., \& Laschke, M. W. 2017. Current and emerging vascularization strategies in skin tissue engineering. Critical Reviews in Biotechnology 37(50): 623-625.

Gardner, S. E., Hillis, S. L., Heilmann, K., Segre, J. A., \& Grice, E. A. 2013. The neuropathic diabetic foot ulcer microbiome is associated with clinical factors. Diabetes 62(3): 923-930.

Garrett, T. R., Bhakoo, M., \& Zhang, Z. 2008. Bacterial adhesion and biofilms on surfaces. Progress in Natural Science 18(9): 1049-1056.

Geske, G. D., Wezeman, R. J., Siegel, A. P., \& Blackwell, H. E. 2005. Small molecule inhibitors of bacterial quorum sensing and biofilm formation. Journal of the American Chemical Society 127(37): 12762-12763.

Gonzalez, F. C., Alramadan, M., Matesanz, M., Diaz, A., Gonzalez-Romo, F., Candel, I. \& Picazo, J. J. 2003. Infections in diabetic foot ulcers. European Journal of Internal Medicine 14(5): 341-343.

Granov, D., Bekic, D., Dulic, E. J., \& Dedeic-Ljubovic, A. 2019. Prevalence and susceptibility pattern of anaerobic bacteria isolated from wound swabs in Clinical Centre University of Sarajevo. Acta Medica Saliniana 49:78-79.

Green, B. 2012. Understanding infection in wound care: wound care. Professional Nursing Today 16(2): 48-55.

Hense, B. A., \& Schuster, M. 2015. Core principles of bacterial autoinducer systems. Microbiology and Molecular Biology Reviews 79(1): 153-169.

Hilton, J. R., Williams, D. T., Beuker, B., Miller, D. R., \& Harding, K. G. 2004. Wound dressings in diabetic foot disease. Clinical Infectious Diseases 39(2): 100-103.

Hirsch, T., Spielmann, M., Zuhaili, B., Koehler, T., Fossum, M., Steinau, H. U., \& Eriksson, E. 2008. Enhanced susceptibility to infections in a diabetic wound healing model. BMC Surgery 8(1): 5-12.

Howell-Jones, R. S., Wilson, M. J., Hill, K. E., Howard, A. J., Price, P. E., \& Thomas, D. W. 2005. A review of the microbiology, antibiotic usage and resistance in chronic skin wounds. Journal of Antimicrobial Chemotherapy 55(2): 143-149.

Kalishwaralal, K., BarathManiKanth, S., Pandian, S. R. K., Deepak, V., \& Gurunathan, S. 2010. Silver nanoparticles impede the biofilm formation by Pseudomonas aeruginosa and Staphylococcus epidermidis. Colloids and Surfaces B. Biointerfaces 79(2): 340-344.

Kostenko, V., Lyczak, J., Turner, K., \& Martinuzzi, R. J. 2010. Impact of silver-containing wound dressings on bacterial biofilm viability and susceptibility to antibiotics during prolonged treatment. Antimicrobial Agents and Chemotherapy 54(12): 5120-5131.

Lalau, J. D., Bresson, R., Charpentier, P., Coliche, V., Erlher, S., Van, G. H., \& Rigal, F. 2002. Efficacy and tolerance of calcium alginate versus vaseline gauze dressings in the treatment of diabetic foot lesions. Diabetes and Metabolism 28(3): 223-229.

Langer, A., \& Rogowski, W. 2009. Systematic review of economic evaluations of human cell-derived wound care products for the treatment of venous leg and diabetic foot ulcers. $B M C$ Health Services Research 9(1): 115-129.

Lau, G. W., Hassett, D. J., Ran, H., \& Kong, F. 2004. The role of pyocyanin in Pseudomonas aeruginosa infection. Trends in Molecular Medicine 10(12): 599-606.

Limoli, D. H., Jones, C. J., \& Wozniak, D. J. 2015. Bacterial extracellular polysaccharides in biofilm formation and function. Microbiology Spectrum 3(3): 1-30.

Martin, J. M., Zenilman, J. M., \& Lazarus, G. S. 2010. Molecular microbiology: new dimensions for cutaneous biology and wound healing. Journal of Investigative Dermatology 130(1): 3848.

Monroe, D. 2007. Looking for chinks in the armor of bacterial biofilms. PLoS Biology 5(11): 2458-2461.

Moody, A. 2006. Use of a hydrogel dressing for management of a painful leg ulcer. British Journal of Community Nursing 11(3): 12-17.

Moura, L. I., Dias, A. M., Carvalho, E., \& de Sousa, H. C. 2013. Recent advances on the development of wound dressings for diabetic foot ulcer treatment-a review. Acta Biomaterialia 9(7): 7093-7114.

Murphy, P. S., \& Evans, G. R. 2012. Advances in wound healing: a review of current wound healing products. Plastic Surgery International 2012: 190436.

Nair, N., Biswas, R., Götz, F., \& Biswas, L. 2014. Impact of Staphylococcus aureus on pathogenesis in polymicrobial infections. Infection and Immunity 82(6): 2162-2169.

Noor, S., Khan, R. U., \& Ahmad, J. 2017. Understanding diabetic foot infection and its management. Diabetes and Metabolic Syndrome: Clinical Research and Reviews 11(2): 149-156.

Oliveira, A., Simões, S., Ascenso, A., \& Reis, C. P. 2020. Therapeutic advances in wound healing. Journal of Dermatological Treatment: 1-21.

O'toole, G., Kaplan, H. B., \& Kolter, R. 2000. Biofilm formation as microbial development. Annual Reviews in Microbiology 54(1): 49-79.

Pallela, S. R. N., \& Narahari, P. 2017. A study to find the causes of diabetic foot infections in a selected community. International Surgery Journal 4(7): 2153-2156.

Parsa, H., \& Samani, S. 2015. Microbiological features and risk factors in patients with diabetic foot ulcers. Wounds: $A$ Compendium of Clinical Research and Practice 27(11): 308-312.

Pastar, I., Nusbaum, A. G., Gil, J., Patel, S. B., Chen, J., Valdes, J., \& Davis, S. C. 2013. Interactions of methicillin resistant Staphylococcus aureus USA300 and Pseudomonas aeruginosa in polymicrobial wound infection. PloS One 8(2): 1-11.

Pednekar, S.N., Pol, S.S., Kamble, S.S., \& Deshpande, S. K. 2015. Drug resistant anaerobic infections: Are they complicating diabetic foot ulcer? International Journal of Healthcare and Biomedical Research 3(3): 142-148.

Percival, S. L., McCarty, S. M., \& Lipsky, B. 2015. Biofilms and wounds: an overview of the evidence. Advances in Wound Care 4(7): 373-381.

Peters, B. M., Jabra-Rizk, M. A., Graeme, A. O., Costerton, J. W., \& Shirtliff, M. E. 2012. Polymicrobial interactions: impact on pathogenesis and human disease. Clinical Microbiology Reviews 25(1): 193-213.

Poon, V. K., \& Burd, A. 2004. In vitro cytotoxity of silver: implication for clinical wound care. Burns 30: 140-147.

Ravari, H., Modaghegh, M. H. S., Kazemzadeh, G. H., Johari, H. G., Vatanchi, A. M., Sangaki, A., \& Shahrodi, M. V. 2013. Comparision of vacuum-asisted closure and moist wound dressing in the treatment of diabetic foot ulcers. Journal of Cutaneous and Aesthetic Surgery 6(1): 17-20.

Renner, L. D., \& Weibel, D. B. 2011. Physicochemical regulation of biofilm formation. Materials Research Society Bulletin 36(5): 347-355.

Rovira, J., Martí N., Marta S., \& José L.D. 2015. Human exposure 
to trace elements through the skin by direct contact with clothing: Risk assessment. Environmental Research 140: 308316.

Sadikot, R. T., Blackwell, T. S., Christman, J. W., \& Prince, A. S. 2005. Pathogen-host interactions in Pseudomonas aeruginosa pneumonia. American Journal of Respiratory and Critical Care Medicine 171(11): 1209-1223.

Selva O. A., Solà, I., Barajas-Nava, L. A., Gianneo, O. D., BonfillCosp, X., \& Lipsky, B. A. 2015. Systemic antibiotics for treating diabetic foot infections. The Cochrane Library 4(9): 118-121.

Senneville, E., Melliez, H., Beltrand, E., Legout, L., Valette, M., Cazaubie, M., \& Mouton, Y. 2006. Culture of percutaneous bone biopsy specimens for diagnosis of diabetic foot osteomyelitis: concordance with ulcer swab cultures. Clinical Infectious Diseases 42(1): 57-62.

Serra, R., Grande, R., Butrico, L., Rossi, A., Settimio, U. F., Caroleo, B., \& de Franciscis, S. 2015. Chronic wound infections: the role of Pseudomonas aeruginosa and Staphylococcus aureus. Expert Review of Anti-infective Therapy 13(5): 605-613.

Short, F. L., Murdoch, S. L., \& Ryan, R. P. 2014. Polybacterial human disease: the ills of social networking. Trends in Microbiology 22(9): 508-516.

Stashak, T. S., Farstvedt, E., \& Othic, A. 2004. Update on wound dressings: indications and best use. Clinical Techniques in Equine Practice 3(2): 148-163.

Tipton, C. D., Sanford, N. E., Everett, J. A., Gabrilska, R. A., Wolcott, R. D., Rumbaugh, K. P., \& Phillips, C. D. 2019. Chronic wound microbiome colonization on mouse model following cryogenic preservation. PloS ONE 14(8):142-147.

Van Netten, J. J., Bus, S. A., Apelqvist, J., Lipsky, B. A., Hinchliffe, R. J., Game, F., \& Senneville, E. 2019. Definitions and criteria for diabetic foot disease. Diabetes/Metabolism Research and Reviens 7: $\mathrm{e} 3268$.

Venault, A., Bai, Y. W., Dizon, G. V., Chou, H. Y. E., Chiang, H. C., Lo, C. T., \& Chang, Y. 2019. Healing kinetics of diabetic wounds controlled with charge-biased hydrogel dressings. Journal of Materials Chemistry B 7(45): 7184-7194.

Wei, D., Zhu, X. M., Chen, Y. Y., Li, X. Y., Chen, Y. P., Liu, H. Y., \& Zhang, M. 2019. Chronic wound biofilms: diagnosis and therapeutic strategies. Chinese Medical Journal 132(22): 2737-2744.

Wei, H., Cheng, S.-X., Zhang, X.-Z., \& Zhuo, R.-X. 2009. Thermo-sensitive polymeric micelles based on poly $(\mathrm{N}$ isopropylacrylamide) as drug carriers. Progress in Polymer Science 34: 893-910

Weledji, E. P., \& Fokam, P. 2014. Treatment of the diabetic foot - to amputate or not? BMC Surgery 14: 1-6.

Weller, C. D., Team, V., \& Sussman, G. 2020. First-Line Interactive Wound Dressing Update: A Comprehensive Review of the Evidence. Frontiers in Pharmacology 11: 155.

Wu, Y. K., Cheng, N. C., \& Cheng, C. M. 2019. Biofilms in chronic wounds: pathogenesis and diagnosis. Trends in Biotechnology 37(5): 505-517.

Yang, W., Lu, J., Weng, J., Jia, W., Ji, L., Xiao, J., \& Zhu, D. 2010. Prevalence of diabetes among men and women in China. New England Journal of Medicine 362(12): 1090-1101.

Zhang, W., Wang, Y., Lee, O. O., Tian, R., Cao, H., Gao, Z., \& Qian, P.-Y. 2013. Adaptation of intertidal biofilm communities is driven by metal ion and oxidative stresses. Scientific Reports 3: 3180-3187.

Zhao, G., Hochwalt, P. C., Usui, M. L., Underwood, R. A., Singh, P. K., James, G. A., \& Olerud, J. E. 2010. Delayed wound healing in diabetic $(\mathrm{db} / \mathrm{db})$ mice with Psendomonas aeruginosa biofilm challenge: a model for the study of chronic wounds. Wound Repair and Regeneration 18(5): 467-477.

Zweiker, D., Horn, S., Hoell, A., Seitz, S., Walter, D., \& Trop, M.
2014. Semi-permanent skin staining associated with silvercoated wound dressing Acticoat. Annals of Burns and Fire Disasters 27(4): 197-200. 